\title{
Myocardial iron overload by cardiovascular magnetic resonance native segmental T1 mapping: a sensitive approach that correlates with cardiac complications
}

\author{
Antonella Meloni ${ }^{1}$, Nicola Martini ${ }^{1}$, Vincenzo Positano ${ }^{1}$, Antonio De Luca ${ }^{2}$, Laura Pistoia', Sara Sbragi ${ }^{3}$, \\ Anna Spasiano ${ }^{4}$, Tommaso Casini ${ }^{5}$, Pier Paolo Bitti ${ }^{6}$, Massimo Allò ${ }^{7}$, Paola Maria Grazia Sanna ${ }^{8}$, \\ Raffaele De Caterina ${ }^{3}$, Gianfranco Sinagra ${ }^{2}$ and Alessia Pepe ${ }^{1 *}$ (D)
}

\begin{abstract}
Background: We compared cardiovascular magnetic resonance segmental native T1 against T2* values for the detection of myocardial iron overload $(\mathrm{MIO})$ in thalassaemia major and we evaluated the clinical correlates of native T1 measurements.

Methods: We considered 146 patients (87 females, 38.7 \pm 11.1 years) consecutively enrolled in the Extension-Myocardial Iron Overload in Thalassaemia Network.

$\mathrm{T} 1$ and $\mathrm{T} 2 *$ values were obtained in the 16 left ventricular (LV) segments. LV function parameters were quantified by cine images. Post-contrast late gadolinium enhancement (LGE) and T1 images were acquired.

Results: $64.1 \%$ of segments had normal T2* and T1 values while $10.1 \%$ had pathologic T2* and T1 values. In 526 (23.0\%) segments, there was a pathologic T1 and a normal T2* value while 65 (2.8\%) segments had a pathologic T2* value but a normal T1 and an extracellular volume (ECV) $\geq 25 \%$ was detected in 16 of 19 segments where ECV was quantified.

Global native T1 was independent from gender or LV function but decreased with increasing age. Patients with replacement myocardial fibrosis had significantly lower native global T1. Patients with cardiac complications had significantly lower native global T1.
\end{abstract}

Conclusions: The combined use of both segmental native $\mathrm{T} 1$ and $\mathrm{T} 2{ }^{*}$ values could improve the sensitivity for detecting MIO. Native T1 is associated with cardiac complications in thalassaemia major.

Keywords: Iron overload, Thalassaemia major, Magnetic resonance imaging, Cardiovascular diseases

*Correspondence: alessia.pepe@ftgm.it

1 Magnetic Resonance Imaging Unit, Fondazione G. Monasterio CNRRegione Toscana, Pisa, Italy

Full list of author information is available at the end of the article

\begin{abstract}
Background
Iron-induced cardiomyopathy is the leading cause of death in $\beta$-thalassaemia major ( $\beta$-TM) patients [1]. Myocardial iron overload (MIO) can be removed or prevented by chelation therapy [2], which should be tailored to the individual patient needs. Thus, techniques for assessing $\mathrm{MIO}$ and its response to chelation therapy are essential for the best management of $\beta$-TM patients.
\end{abstract} permits use, sharing, adaptation, distribution and reproduction in any medium or format, as long as you give appropriate credit to the original author(s) and the source, provide a link to the Creative Commons licence, and indicate if changes were made. The images or other third party material in this article are included in the article's Creative Commons licence, unless indicated otherwise in a credit line to the material. If material is not included in the article's Creative Commons licence and your intended use is not permitted by statutory regulation or exceeds the permitted use, you will need to obtain permission directly from the copyright holder. To view a copy of this licence, visit http://creativecommons.org/licenses/by/4.0/. The Creative Commons Public Domain Dedication waiver (http://creativeco mmons.org/publicdomain/zero/1.0/) applies to the data made available in this article, unless otherwise stated in a credit line to the data. 
To date, cardiovascular magnetic resonance (CMR) is the only non-invasive technique for quantification of MIO. The presence of myocardial iron deposits causes microscopic magnetic field inhomogeneities and results in a reduction in $\mathrm{T} 1, \mathrm{~T} 2$ and $\mathrm{T} 2 *$ relaxation times [3]. The T2* technique, introduced in 1999 [4], is currently the method of choice for cardiac iron quantification and it represents the first parametric mapping technique to become a clinical tool and a reference method according to clinical guidelines [5]. The $\mathrm{T}^{*}$ technique is fast, robust, reproducible, transferable among different CMR scanners [6-8], and validated against tissue in human models $[9,10]$. The deployment of $\mathrm{T} 2$ * CMR has been recognized as one of the main drivers for the improvement in survival of TM patients, leading to the reduction in deaths due to MIO [1]. In fact, cardiac $\mathrm{T} 2$ * values have a prognostic role, allowing the early identification of patients at risk for heart failure and needing an intensification of chelation therapy [11, 12]. However, the $\mathrm{T} 2 *$ technique is sensitive to susceptibility artifacts and has reduced sensitivity for detection of changes associated with mild or early MIO [13].

In vivo mapping of the myocardial $\mathrm{T} 1$ relaxation time has been proposed as a possible approach to overcome this limitation. A significant correlation between midseptum native $\mathrm{T} 1$ and $\mathrm{T} 2 *$ values has been detected in patients with $\beta$-TM and other haemoglobinopathies [14-18].

No literature data are available about the association between native $\mathrm{T} 1$ and $\mathrm{T} 2 *$ using a segmental approach including the entire left ventricle (LV). As previously demonstrated for the $\mathrm{T}_{2} *$ technique, the segmental approach is more sensitive, allowing to detect MIO in the various cardiac segments before it occurs at the septal level [19]. This approach is particularly valuable in young patients [20] and allows the detection of preferential patterns of iron distribution correlated with clinical endpoints [12, 21].

To date, no study has evaluated whether T1 measurements quantifying MIO are influenced by age, gender, or myocardial fibrosis. Of note, replacement myocardial fibrosis detected by late gadolinium enhancement (LGE) CMR is common in $\beta$-TM patients [22] and it is associated with an higher risk of cardiac complications [12]. By preliminary experience extracellular volume (ECV) appears to be increased in TM and associated with MIO [23]. Finally, the clinical impact of T1 for detecting cardiac complications in $\beta$-TM has never been explored.

We sought to assess the relationship between native $\mathrm{T} 1$ and $\mathrm{T} 2 *$ values using a segmental approach, as well as the association between global T1 measurements, demographics, and cardiac involvement.

\section{Methods}

\section{Study population}

We considered $146 \beta$-TM patients (87 females, $38.7 \pm 11.1$ years), consecutively enrolled in the Extension-Myocardial Iron Overload in Thalassaemia (E-MIOT) project, an Italian network constituted by 66 thalassaemia centres and 11 validated CMR sites [8, 24, 25] sharing clinical data.

The study complied with the Declaration of Helsinki and was approved by the local ethical committee. All patients gave written informed consent.

\section{CMR protocol}

CMR exams were performed in the reference CMR center of the E-MIOT Network (Pisa) using a $1.5 \mathrm{~T}$ CMR scanner (Signa Artist; General Electric Healthcare, Waukesha, Wisconsin, USA). A 30-element cardiac phased-array receiver surface coil with breath-holding and electrocardiographic (ECG)-gating was used.

For T1 mapping, three parallel short-axis slices (basal, medium and apical) of the LV were acquired in end-diastole using a modified Look-Locker inversion recovery (MOLLI) sequence [26] with a 3 (3 s) 3 (3 s) 5 scheme. Other sequence parameters were: slice thickness $=8 \mathrm{~mm}$, flip angle $=35^{\circ}$, matrix $=172 \times 172$ pixels, partial Fourier $=0.75$. Starting from the 11 motion-corrected images with different inversion times, pixel-wise T1 maps were generated on the scanner using a three-parameter fitting model [26]. T1 maps were transferred to a dedicated workstation for offline post-processing, that involved manual tracing of endocardial and epicardial borders, with care taken to avoid blood pool and epicardial fat. Basal and medium slices were divided into 6 equiangular segments and the apical slice in 4 segments, according to the American Heart Association/American College of Cardiology model [27]. The T1 in each segment was obtained by averaging the $\mathrm{T} 1$ value for all the pixels within the segment. For blood analysis, a region was drawn in the LV blood pool of each short axis slice avoiding papillary muscles.

$\mathrm{T} 2 *$ multiecho images were acquired in the same 3 slice locations used for $\mathrm{T} 1$ mapping by a multi-echo gradient-echo $\mathrm{T} 2 *$ sequence (10 echo times-TEs). Sequence parameters and $\mathrm{T} 2 *$ images analysis, performed using a custom-written and validated software (HIPPOMIOT $\left.{ }^{\circledR}\right)$, have been already described [19]. After the calculation of $\mathrm{T}_{2} *$ values in all myocardial segments, an appropriate correction map compensated for cardiac/visceral geometrical and susceptibility artifacts [28].

Global $\mathrm{T} 1$ and $\mathrm{T} 2 *$ values were obtained by averaging all segmental $\mathrm{T} 1$ and $\mathrm{T} 2 *$ values, respectively. Mid-septum $\mathrm{T} 1$ and $\mathrm{T} 2 \%$ values were obtained by averaging the values in segments 8 and 9 . 
Balanced steady-state free precession cine images were acquired in sequential 8-mm short-axis slices from the atrio-ventricular ring to the apex to quantify LV function parameters in a standard way using MASS ${ }^{\circledR}$ software (Medis, Leiden, The Netherlands) [29]. LV volumes and mass were normalized for the body surface area. Segmental wall motion was visually assessed in cine images.

To detect the presence of replacement myocardial fibrosis, LGE short-axis, vertical, horizontal, and oblique long-axis images were acquired 10-18 min after gadobutrol (Gadovist ${ }^{\circledR}$; Bayer Healthcare; Berlin, Germany) intravenous administration $(0.2 \mathrm{mmoL} / \mathrm{kg})$. LGE images were not acquired in patients with an estimated glomerular filtration rate $<30 \mathrm{~mL} / \mathrm{min} / 1.73 \mathrm{~m}^{2}$ and in patients who declined contrast. LGE was considered present when visualized in two different views [22,30].

Post-contrast T1 images were acquired $10 \mathrm{~min}$ after contrast medium administration and image analysis was performed by using the same approach employed for native T1 images. Segmental ECV values were calculated with input of native and post-contrast myocardial segmental and blood pool T1 values and same-day hematocrit [31].

\section{Diagnostic criteria}

The lower limit of normal T1 and T2* for each segment was established as mean-2 standard deviations (SD) on data acquired on 80 healthy subjects. Since in healthy subjects $\mathrm{T} 1$ values are influenced by gender, gender-specific segmental thresholds were derived.

Heart failure (HF) was diagnosed by clinicians based on symptoms, signs and instrumental findings [32]. All arrhythmias were ECG-documented and required specific medication [33]. The term "cardiac complications" included HF and arrhythmias.

\section{Reproducibility analysis for $\mathrm{T} 1$ measurements}

To evaluate the intra-observer reproducibility, native $\mathrm{T} 1$ images from 20 patients were re-analyzed by the same operator after 4 weeks. To evaluate the inter-observer reproducibility, the same images were blindly analyzed by a second operator. The coefficient of variation $(\mathrm{CoV})$ was calculated as the ratio of the SD to the mean, multiplied by 100 . The intraclass correlation coefficient (ICC) was obtained from a two-way random effects model with measures of absolute agreement. The Bland-Altman technique was used to plot the absolute difference versus the average values between the two datasets. Bias was the mean of the difference between the two sets of values and agreement was the mean \pm 1.96 SDs.

\section{Statistical analysis}

All data were analyzed using SPSS (version 18.0, Statistical Package for the Social Sciences, International Business Machines, Inc., Armonk, New York, USA).

Continuous variables were described as mean $\pm \mathrm{SD}$ and categorical variables were expressed as frequencies and percentages.

The Kolomogorov-Smirnov test showed a non-normal distribution for T1 values.

The comparison of $\mathrm{T} 1$ values between two groups was made by the Wilcoxon rank sum test. $\chi^{2}$ testing was performed for non-continuous variables.

Correlation analysis was performed using the Spearman's test.

Logistic regression was used to evaluate the odds ratio (OR) with 95\% confidence intervals (CI). The OR was used to compare the odds for two groups.

To determine the best cut-off of $\mathrm{T} 1$ or $\mathrm{T} 2 *$ values for discriminating the presence of a specific condition, the maximum sum of sensitivity and specificity was calculated from receiver-operating characteristic (ROC) curve analysis.

A 2-tailed $\mathrm{P}<0.05$ was considered statistically significant.

\section{Results}

\section{Patients' characteristics}

Patients' demographic and clinical characteristics are summarized in Table 1.

Table 1 Demographic, clinical and cardiovascular magnetic resonance (CMR) data of the patients

\begin{tabular}{ll}
\hline Variable & Value \\
\hline Sex (males/females) & $59 / 87$ \\
Age (years) & $38.7 \pm 11.1$ \\
Transfusion starting age (months) & $16.5 \pm 14.9$ \\
Chelation starting age (years) & $4.5 \pm 5.6$ \\
Splenectomy, \% & 40.6 \\
Past or active hepatitis C virus infection, (\%) & 67.2 \\
Pre-transfusion hemoglobin (g/dl) & $9.7 \pm 0.4$ \\
Serum ferritin (ng/l) & $1205 \pm 1147$ \\
Global myocardial T1 (ms) & $952 \pm 99$ \\
Global myocardialT2*(ms) & $37.4 \pm 9.3$ \\
LV end-diastolic volume index (ml/m²) & $83.8 \pm 16.4$ \\
LV end-systolic volume index (ml/m²) & $31.7 \pm 10.3$ \\
LV stroke volume index (ml/m²) & $52.0 \pm 9.9$ \\
LV mass index & $59.6 \pm 12.8$ \\
LV ejection fraction (\%) & $62.5 \pm 7.4$ \\
Abnormal LV motion, N (\%) & $14(9.6)$ \\
Replacement myocardial fibrosis, N (\%) & $36 / 88(40.9 \%)$ \\
\hline
\end{tabular}

$L V$ left ventricular, $N$ number 
Mean global heart T1 value was $952 \pm 99 \mathrm{~ms}$ (range 494-1170 ms) while mean global heart $\mathrm{T} 2$ * value was $37.4 \pm 9.3 \mathrm{~ms}$ (range $5.2-37.4 \mathrm{~ms}$ ).

Figure 1 shows $\mathrm{T} 1$ and $\mathrm{T} 2 *$ reports for one patient.

\section{Corrrelation between global heart and mid-septum T1 and T2* values $^{*}$}

A significant correlation was detected between global heart and mid-septum T1 values $(\mathrm{R}=0.914 ; \mathrm{P}<0.001)$. Out of the 70 patients with a reduced global heart $\mathrm{T} 1$ value $(<928 \mathrm{~ms}$ in males and $<989 \mathrm{~ms}$ in females), $58(82.0 \%)$ had also a reduced mid-septum T1 value (<919 $\mathrm{ms}$ in males and $<966 \mathrm{~ms}$ in females) while 12 (17.1\%) had a normal mid-septum T1 value. Furthermore, out of the 85 patients with a normal mid-septum $\mathrm{T} 1$ value, 44 (51.8\%) showed a pathological $\mathrm{T} 1$ value in at least one of the remaining 14 segments. For three patients a reduced mid-septum $\mathrm{T} 1$ value was detected in presence of a normal global heart $\mathrm{T} 1$ value, but all of them had also other two segments with reduced $\mathrm{T} 1$ value.

For $\mathrm{T}^{*}$ a significant correlation was detected between global heart and mid-septum values $(\mathrm{R}=0.733$; $\mathrm{P}<0.001)$. Four patients had a normal mid-septum $\mathrm{T} 2 *$ value in presence of a reduced global heart $\mathrm{T} 2 *$ value and out of the 127 patients with a normal mid-septum T2* value, 35 (27.6\%) showed a pathological $\mathrm{T} 1$ value in at least one of the remaining 14 segments.

\section{Intra- and inter-observer variability}

The results of the intra- and inter-observer variability analysis for segmental and global $\mathrm{T} 1$ values are indicated in Table 2. The CoV was below 5\% for all segments and the ICC was always excellent $(>0.90)$. The Bland-Altman analysis demonstrated no significant bias.

\section{Correlation between $\mathrm{T} 1$ and $\mathrm{T} 2 *$ values by a segmental approach}

T1 images showed more pronounced motion artifacts and lower contrast-to-noise-ratio, determining the exclusion of 49/2336 (2.1\%) segments. No segments were excluded in T2* images. Globally 2287 segmental T1 and $\mathrm{T}^{*}$ values were considered.

Figure 2 shows the relationship between segmental T1 and $\mathrm{T} 2 *$ values. For patients with pathologic segmental $\mathrm{T}^{*}$ values there was a linear relationship between segmental T1 and T2* values $(\mathrm{R}=0.775, \mathrm{P}<0.001)$. Normal $\mathrm{T}^{*}$ and $\mathrm{T} 1$ values were found in $465(64.1 \%)$ segments while $231(10.1 \%)$ segments had pathologic $\mathrm{T} 2 *$ and $\mathrm{T} 1$ values, giving a concordance between the two measurements of $74.2 \%$. For 526 (23.0\%) segments (95 patients) a pathologic $\mathrm{T} 1$ value was detected in the presence of a normal T2* value. For 65 (2.8\%) segments (33 patients) a pathologic $\mathrm{T} 2 *$ value was detected in the presence of a normal T1 value. ECV was measured in 19 of 65 segments with pathologic T2* but normal T1 value (12 patients) and an ECV $\geq 25 \%$ was detected in 16 (84.2\%) segments. LGE images were available for 30/65 segments (18 patients) and only one segment showed LGE-positivity. This single segment had also increased ECV.

A significant correlation was detected between global heart $\mathrm{T} 1$ and $\mathrm{T} 2 *$ values $(\mathrm{R}=0.449 ; \mathrm{P}<0.001)$. Out of the $22(15.1 \%)$ patients with a pathologic global heart T2* value $(<32 \mathrm{~ms})$, two had a normal T1. Fifty $(34.2 \%)$ patients had a pathologic global T1 in the presence of a normal global T2*.

\section{Global T1 and demographics}

Myocardial global T1 was comparable between males and females ( $944 \pm 90$ vs. $957 \pm 104 \mathrm{~ms} ; \mathrm{P}=0.073)$ while they showed a weak inverse correlation with age $(R=-0.220$; $\mathrm{P}=0.007)$.

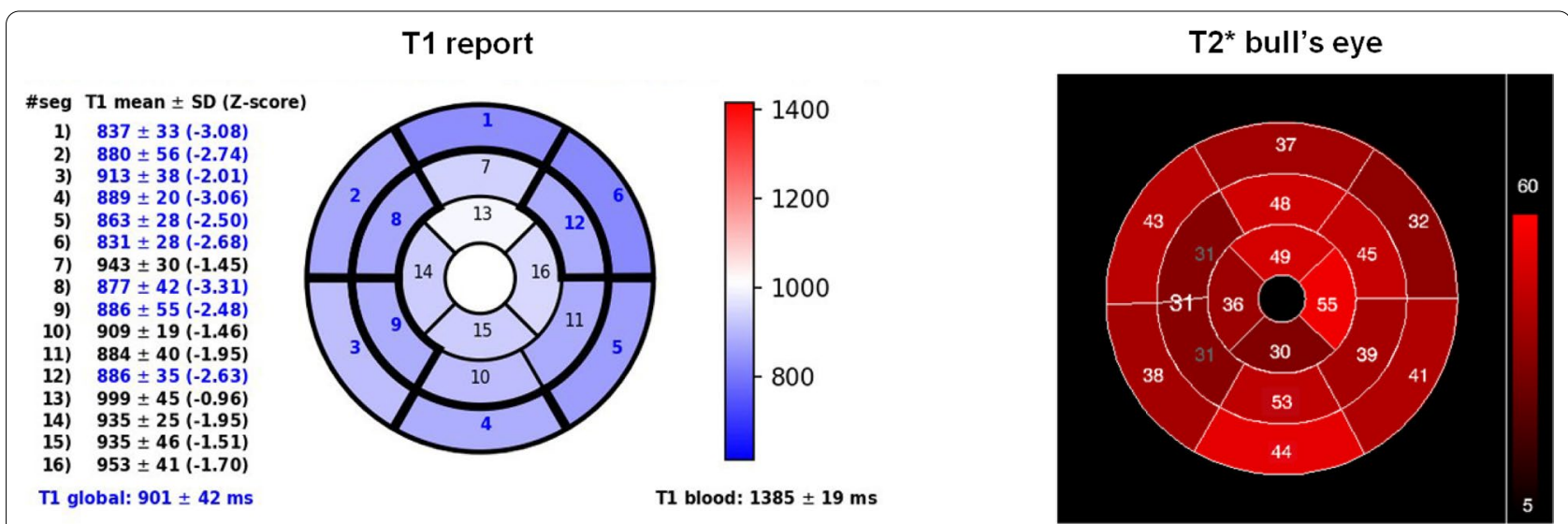

Fig. 1 Representative myocardial native T1 and T2* reports for a patient with pathologic segmental T1 but normal T2* 
Table 2 Intra- and inter-observer reproducibility data for T1 measurements

\begin{tabular}{|c|c|c|c|c|c|c|c|c|}
\hline & \multicolumn{4}{|c|}{ Intra-observer } & \multicolumn{4}{|c|}{ Inter-observer } \\
\hline & \multirow[t]{2}{*}{$\operatorname{CoV}(\%)$} & \multirow[t]{2}{*}{ ICC $(95 \% \mathrm{Cl})$} & \multicolumn{2}{|c|}{ Bland-Altman analysis } & \multirow[t]{2}{*}{$\operatorname{CoV}(\%)$} & \multirow[t]{2}{*}{ ICC $(95 \%$ CI) } & \multicolumn{2}{|c|}{ Bland-Altman analysis } \\
\hline & & & Bias (ms) & Limits (ms) & & & Bias (ms) & Limits (ms) \\
\hline Segment 1 & 1.45 & 0.997 (0.993-0.999) & -2.7 & $-35.6-39.2$ & 4.83 & 0.996 (0.991-0.999) & 4.6 & $-33.1-42.2$ \\
\hline Segment 2 & 2.24 & $0.992(0.979-0.997)$ & 1.7 & $-51.5-54.8$ & 2.09 & $0.992(0.980-0.997)$ & 9.9 & $-36.1-55.8$ \\
\hline Segment 3 & 1.17 & 0.998 (0.994-0.999) & 3.3 & $-23.7-30.4$ & 2.42 & $0.991(0.977-0.996)$ & 5.6 & $-50.9-62.2$ \\
\hline Segment 4 & 1.62 & 0.996 (0.989-0.998) & 0.1 & $-39.1-39.3$ & 1.82 & 0.994 (0.984-0.998) & 9.8 & $-29.7-49.3$ \\
\hline Segment 5 & 1.45 & 0.995 (0.987-0.998) & -4.1 & $-37.9-29.7$ & 3.10 & $0.977(0.942-0.991)$ & 8.4 & $-64.3-81.1$ \\
\hline Segment 6 & 1.81 & $0.994(0.985-0.998)$ & 0.6 & $-41.3-42.5$ & 2.79 & $0.986(0.961-0.995)$ & 13.6 & $-45.5-72.6$ \\
\hline Segment 7 & 2.42 & $0.992(0.981-0.997)$ & 7.6 & $-49.3-64.5$ & 3.83 & 0.979 (0.948-0.992) & 12.7 & $-77.8-103.1$ \\
\hline Segment 8 & 1.38 & 0.997 (0.992-0.999) & -6.9 & $-36.8-23.0$ & 1.79 & $0.995(0.975-0.999)$ & 13.8 & $-19.2-46.9$ \\
\hline Segment 9 & 1.32 & 0.998 (0.994-0.999) & -6.3 & $-34.5-21.9$ & 3.29 & $0.986(0.965-0.995)$ & 14.5 & $-57.4-86.4$ \\
\hline Segment 10 & 1.59 & 0.997 (0.992-0.999) & -5.0 & $-41.4-31.4$ & 2.49 & $0.992(0.981-0.997)$ & 5.9 & $-52.2-63.9$ \\
\hline Segment 11 & 1.77 & $0.9954(0.986-0.998)$ & 5.3 & $-36.7-47.3$ & 1.79 & $0.994(0.985-0.998)$ & -4.7 & $-47.8-38.4$ \\
\hline Segment 12 & 1.74 & 0.994 (0.986-0.998) & 3.7 & $-38.9-46.3$ & 2.93 & 0.984 (0.960-0.994) & 10.2 & $-60.4-80.8$ \\
\hline Segment 13 & 2.50 & $0.985(0.962-0.994)$ & -8.2 & $-75.2-58.9$ & 2.13 & $0.989(0.970-0.996)$ & 11.7 & $-42.2-65.9$ \\
\hline Segment 14 & 1.52 & 0.996 (0.989-0.998) & 2.8 & $-36.3-41.9$ & 2.55 & $0.987(0.964-0.995)$ & 14.9 & $-44.7-74.5$ \\
\hline Segment 15 & 1.90 & 0.994 (0.985-0.998) & 3.0 & $-45.5-51.4$ & 3.19 & $0.983(0.957-0.993)$ & -1.5 & $-83.4-80.4$ \\
\hline Segment 16 & 2.61 & $0.985(0.963-0.994)$ & -0.1 & $-74.0-73.9$ & 2.22 & $0.988(0.971-0.995)$ & -5.7 & $-67.3-56.0$ \\
\hline Global & 0.70 & $0.999(0.998-1.000)$ & -0.3 & $-17.7-17.0$ & 1.35 & 0.997 (0.989-0.999) & 7.7 & $-21.7-37.2$ \\
\hline
\end{tabular}

$\mathrm{CoV}$ coefficient of variation, ICC intraclass correlation coefficient

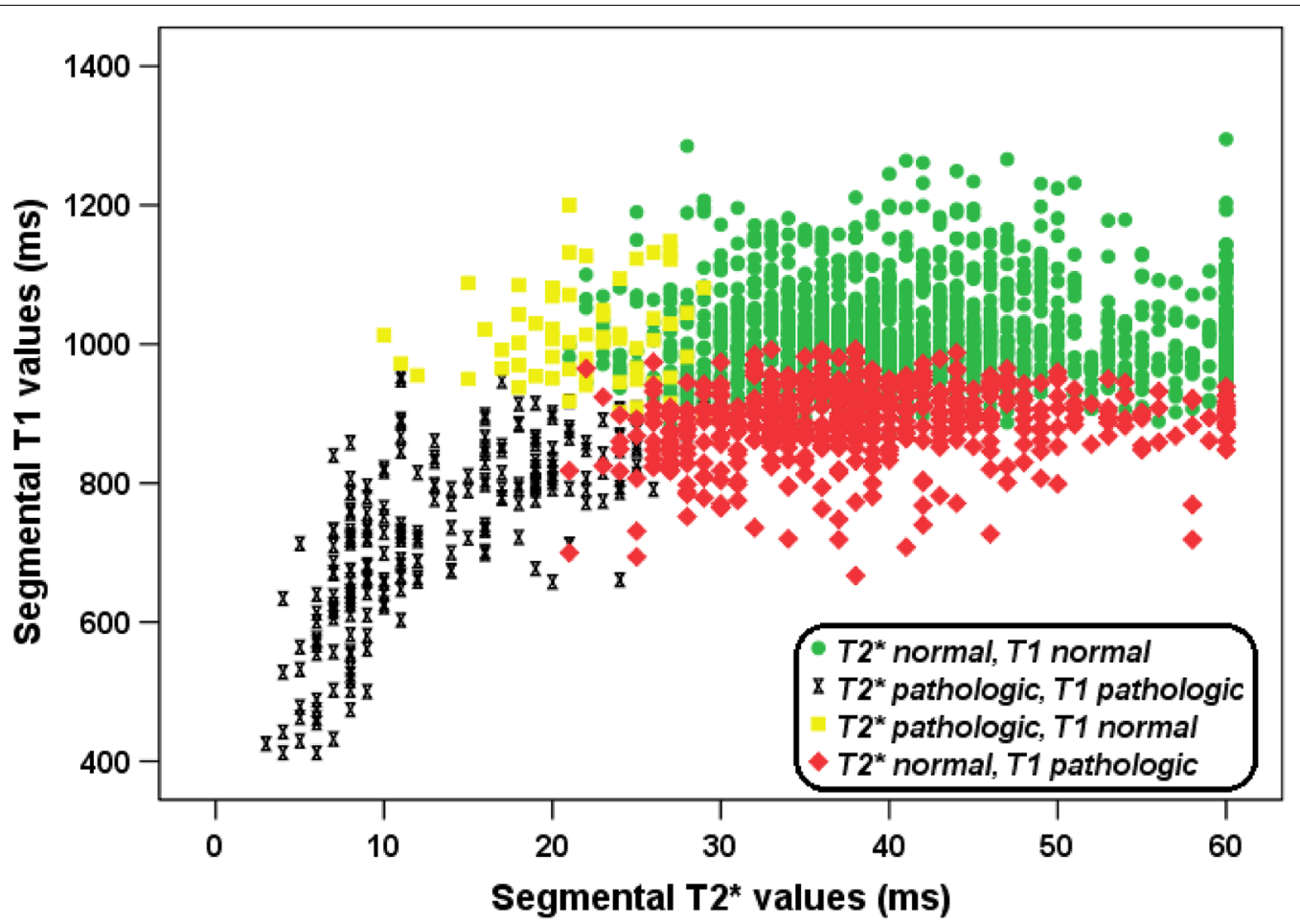

Fig. 2 Scatter plot showing the relationship between segmental $\mathrm{T} 1$ and $\mathrm{T} 2^{*}$ 
A significant inverse correlation was detected between global $\mathrm{T} 1$ and serum ferritin levels $(\mathrm{R}=-0.297$; $\mathrm{P}=0.009$ ).

\section{Global heart T1 values and CMR findings}

No correlation was detected between global T1 and LV volume indexes, mass index, or ejection fraction.

Abnormal LV motion was found in 14 (9.6\%) patients: 13 hypokinetic and one dyskinetic. Patients with abnormal LV motion had significantly lower global T1 than patients without LV motion abnormalities ( $884 \pm 140$ vs. $959 \pm 91 \mathrm{~ms} ; \mathrm{P}=0.049$ ). At ROC curve analysis, a global $\mathrm{T} 1 \leq 865 \mathrm{~ms}$ predicted the presence of LV motion abnormalities with a sensitivity of $42.9 \%$ and a specificity of $90.2 \%(\mathrm{P}=0.049)$. The area under the curve (AUC) was 0.66 (95\% Confidence interval: 0.58-0.74).

LGE images were acquired in 88 (60.3\%) patients; replacement myocardial fibrosis was detected in 36 (40.9\%) patients. Two patients showed an ischemic pattern. Among the patients with LGE, $72.2 \%$ had two or more foci of fibrosis. Patients with replacement myocardial fibrosis had significantly lower global T1 $(921 \pm 100$ vs. $975 \pm 73 \mathrm{~ms} ; \mathrm{P}=0.027$ ) (Fig. $3 \mathrm{a}$ ) and $\mathrm{T} 2 *$ values $(34.2 \pm 11.5 \mathrm{~ms}$ vs. $39.2 \pm 6.9 \mathrm{~ms} ; \mathrm{P}=0.036)$. At $\mathrm{ROC}$ curve analysis, a global heart $\mathrm{T} 1$ value $\leq 898 \mathrm{~ms}$ predicted the presence of positive LGE with a sensitivity of $36.1 \%$ and a specificity of $94.2 \%(\mathrm{P}=0.026)$. The AUC was 0.64 (95\% Confidence interval: 0.53-0.74) (Fig. 3b).

LGE was present for 1408 segments (88 patients x16 segments) and 105 (7.5\%) were positive. Segments with LGE had significantly lower T1 than segments LGE-negative $(906 \pm 111 \mathrm{~ms}$ vs. $957 \pm 104 \mathrm{~ms} ; \mathrm{P}<0.001)$.

\section{$\mathrm{T} 1$ values and cardiac complications}

Nineteen patients had an history of cardiac complications: $11 \mathrm{HF}$ and 8 arrhythmias (7 supraventricular and 1 ventricular). Patients with cardiac complications had significantly lower global T1 ( $874 \pm 122$ vs. $962 \pm 99 \mathrm{~ms}$; $\mathrm{P}<0.001)$ (Fig. 4a) but similar $\mathrm{T}^{*} \quad(33.9 \pm 11.7$ vs. $36.9 \pm 9.3 \mathrm{~ms} ; \mathrm{P}=0.260$ ). Cardiac complications were more frequent in the group of patients with reduced versus normal global T1 (29.8\% vs. 9.1\%; P $=0.010)$. OR for cardiac complications was $4.2(95 \% \mathrm{CI}=1.4-12.9)$ for patients with reduced global $\mathrm{T} 1$ versus patients with normal global T1.

At ROC curve analysis, a global $\mathrm{T} 1 \leq 939 \mathrm{~ms}$ predicted a positive history of cardiac complications with a sensitivity of $78.9 \%$ and a specificity of $72.3 \%$ $(\mathrm{P}<0.001)$. The AUC was 0.77 (95\% confidence interval: $0.68-0.85$ ). The ROC curve did not reveal a global heart $\mathrm{T} 2 *$ threshold below which the probability of detecting a positive history of cardiac complications increases

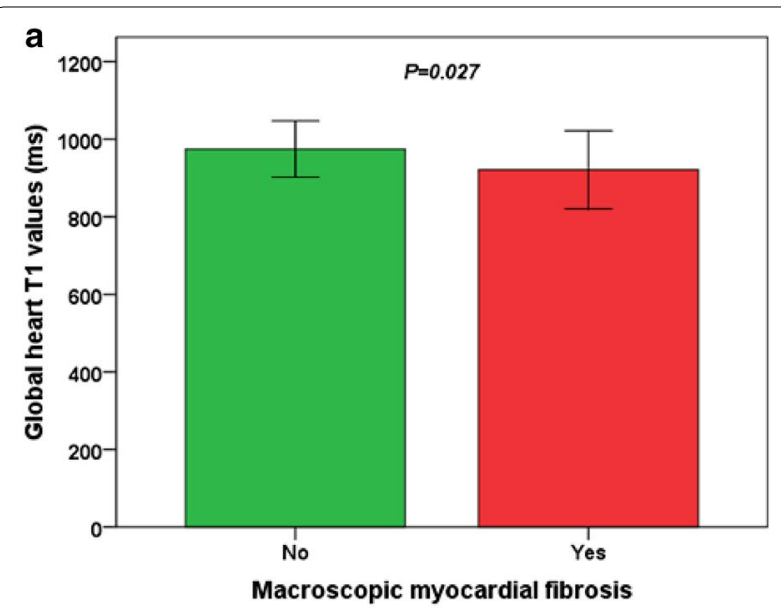

b

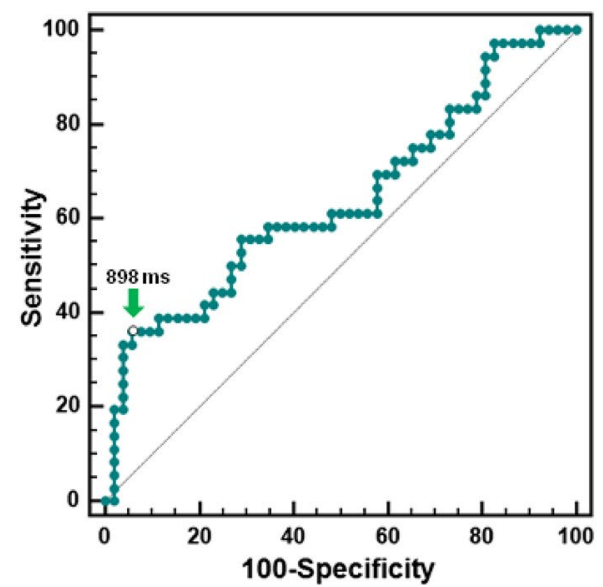

Fig. 3 a Global T1 values in patients without and with replacement myocardial fibrosis detected by late gadolinium enhancement (LGE). b ROC curve analysis of global heart $T 1$ values to predict LGE positivity

significantly with satisfying sensitivity and specificity $(\mathrm{AUC}=0.58 ; \mathrm{P}=0.238$ ). The Delong's test showed a significant difference among the AUCs $(P=0.005)$ (Fig. 4b).

The optimal cut-off for the mid-septum T1 value was $957 \mathrm{~ms}(\mathrm{P}<0.001)$ and the AUC was $0.70(95 \%$ Confidence interval: $0.66-0.83$ ). No significant difference $(P=0.449)$ was obtained between the AUCs obtained for global and mid-septum T1.

For eight patients, the cardiac complication was still active at the time of the CMR (6 supraventricualr arrhythmias and $2 \mathrm{HF}$ ). These patients had a lower T1 than the group of patients without history of cardiac complications or with resolved cardiac complications at the time of CMR $(855 \pm 153$ vs. $954 \pm 101 \mathrm{~ms}$; $\mathrm{P}=0.012$ ) but comparable global heart $\mathrm{T} 2 *$ values $(34.2 \pm 12.3$ vs. $36.6 \pm 9.6 \mathrm{~ms} ; \mathrm{P}=0.422)$. 


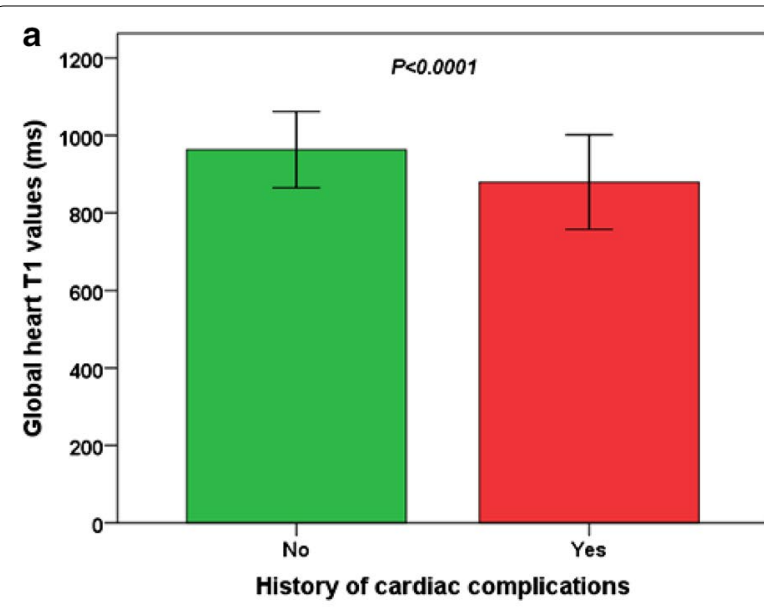

b

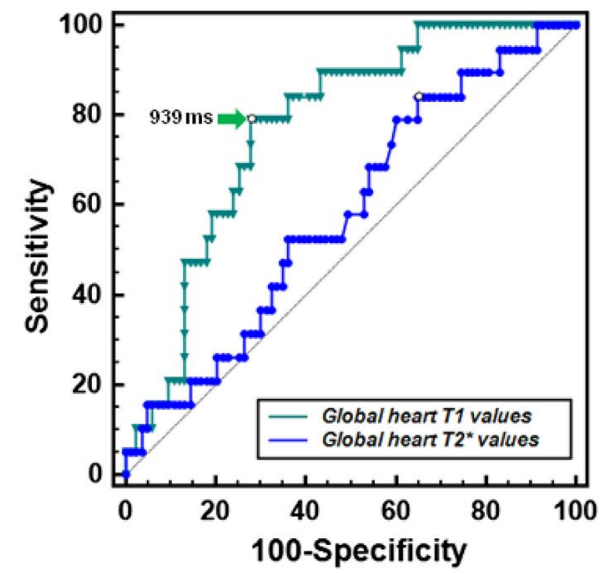

Fig. 4 a Global myocardial $\mathrm{T} 1$ in patients without and with history of cardiac complications. b ROC curve analysis of global T1 (green) and T2* (blue) to identify a positive history of cardiac complications

\section{Discussion}

To our knowledge, this is the first report relating native $\mathrm{T} 1$ to $\mathrm{T} 2 *$ values by a segmental approach including the entire LV wall in haemochromatotic patients. The segmental approach allows to identify a heterogeneous and early iron distribution, that would remain otherwise unexplored with a single measurement in the midventricular septum $[10,19,34]$. For both $\mathrm{T} 1$ and $\mathrm{T} 2 \%$, although we found a significant correlation between global and mid-septum values, the segmental approach was demonstrated to be more sensitive. For T2* mapping the clinical advantages of the more sensitive segmental technique have been demonstrated in order to have an iron-free patient [12, 19-21, 34, 38, 43]. For T1 mapping larger and prospective studies are recommended.

The robustness of the $\mathrm{T}^{*}$ segmental approach had been previously established by proper validation that documented good intra- and inter-observer, inter-study and inter-scanner reproducibility, however decreasing for higher $\mathrm{T} 2$ * values $[8,24]$. The present study showed a superior reproducibility for segmental and global T1 in the whole range of values, although T1 images showed lower contrast-to-noise-ratio.

An association between mid-septum T1 and T2* values at $1.5 \mathrm{~T}$ has been previously shown in relatively large cohorts of $\beta$-TM patients [14-17], and it has been postulated that T1 may provide improved myocardial tissue characterization, due to the presence of a considerable group of patients with normal $\mathrm{T} 2 \%$ but low $\mathrm{T} 1$. However, all published studies used a value of $20 \mathrm{~ms}$ as $\mathrm{T} 2 *$ cutoff, established at an early stage of $\mathrm{T} 2 *$ technique development, using data from only 15 healthy subjects and an obsolete multibreathold gradient-echo technique [4]. This is a conservative cut-off that prioritizes specificity over sensitivity. In the present study, we applied a study-specific cut-off for segmental and global $\mathrm{T} 2 *$ values and the same healthy population was used to derive the lower limits of normal for native T1, highly specific to the local set-up [35].

In the present study, $\mathrm{T}^{*}$ and $\mathrm{T} 1$ showed a good correlation in identifying iron by a segmental approach, with $74 \%$ concordance. In 33 patients, T1 mapping could not detect iron in one or more segments, probably due to the presence of diffuse fibrosis, which is known to increase the native T1 [36]. In fact, an increased ECV, a validated surrogate marker of diffuse fibrosis [37], was detected in 16 out of the 19 discordant segments in which the ECV was available. Unlike T2*, T1 is less specific for iron, and early iron may therefore be missed in patients with diffuse fibrosis, frequent in thalassaemia patients with a history of MIO [23]. A pathologic T1 value was detected in presence of a normal $\mathrm{T} 2 *$ value for 526 segments, suggesting a higher sensitivity of T1 mapping in comparison to the $\mathrm{T}^{*}$ technique in borderline patients. In fact, in this study this discrepancy could not be explained by the use of a low-sensitive $\mathrm{T}^{*}$ threshold or by the presence of susceptibility artifacts, corrected by the use of a validated correction map [28]. Segmental variability due to susceptibility artifacts masks T2* values heterogeneity associated with inhomogeneous iron overload in patients with global $\mathrm{T}^{*}>32 \mathrm{~ms}$ [38]. One of the most plausible explanation for the lower sensitivity of the $\mathrm{T}^{*}$ technique in the detection of mild MIO is that, due to technical constraints (maximum TE), T2* quantification loses accuracy and precision for cardiac $\mathrm{T} 2^{*}$ values $>20 \mathrm{~ms}$ [13]. Moreover, T1 and T2* seem sensitive to different form of iron. Excess iron is first stored within ferritin and, as its concentration increases 
further, it is gathered within hemosiderin. The $\mathrm{T} 2 *$ is predominately determined by hemosiderin [39].

The second objective of our study was to correlate global T1 measurements with demographics, CMR findings, and cardiac complications.

In healthy subjects, native $\mathrm{T} 1$ is influenced by gender, with women having a higher value [40]. In $\beta$-TM patients we detected comparable T1 among males and females, likely because iron masks gender differences of $\mathrm{T} 1$. This finding is in agreement with the $\mathrm{T} 2 *$ studies indicating that males and females are at the same risk of accumulating iron in their hearts [29, 41]. A significant inverse, although weak, association was detected between $\mathrm{T} 1$ and age, suggesting that MIO is an agedependent process as previously demonstrated by the T2* technique [22].

We did not detect any correlation between native T1 and LV systolic function, volumes, or mass, probably because the majority of our patients had normal or mild abnormal values of these parameters, thanks to a good transfusional regimen. Conversely, although not frequent, LV movement abnormalities were associated with low native $\mathrm{T} 1$.

TM patients with replacement myocardial fibrosis had significantly lower T1. The presence of replacement myocardial fibrosis detected by the LGE technique was shown to significantly increase native $\mathrm{T} 1$ [42], even in non-ischemic myocardial diseases. Therefore, our finding has two important implications. Firstly, it suggests the dominance of iron over myocardial fibrosis, likely due to the small percentage of replacement fibrosis within the LV myocardium and its patchy distribution, as previously demonstrated for the T2*-replacement fibrosis relashionship [30]. Second, it suggests an association between $\mathrm{MIO}$ and replacement fibrosis, previously demonstrated in pediatric TM patients [43]. We introduced a threshold of $898 \mathrm{~ms}$ for prediction of LGE with high specificity. Conversely, the low sensitivity of our threshold (about $36 \%$ may reflect as hepatitis $C$ virus infection or diabetes are proved factors in the pathogenesis of replacement myocardial fibrosis in patients with $\beta$-TM $[44,45]$.

Finally, we here demonstrated for the first time an association between decreased native global $\mathrm{T} 1$ and a history of cardiac complications and we introduced a threshhold of $931 \mathrm{~ms}$ for the prediction of cardiac complications with satisfying sensitivity and specificity. Global myocardial $\mathrm{T}^{*}$ was similar among patients without and with cardiac complications. Likely in our population of well treated patients, generally not heavily loaded at the cardiac level, T1 emerge as a more sensitive marker of cardiac complications, stronger than global $\mathrm{T} 2 *$. The comparison of cardiac $\mathrm{T} 1$ values between patients without and with active cardiac complications remained significant despite the low number of patients with active cardiac complications and despite the active complications were represented by supraventricular arrhythmias in $75 \%$ of the cases. MIO was shown to contribute less to the development of supraventricular arrhythmias than to HF [11, 12, 29].

Our findings suggest that, due to its added diagnostic value, native T1 should be included in the routine CMR assessment of $\beta$-TM patients, where available and after the definition of reference ranges for $\mathrm{T} 1$ values in healthy human myocardium. Such a more sensitive approach in quantifying iron could support clinicians in earlier adjustment of chelation therapy and possibly further improve prognosis in $\beta$-TM.

\section{Limitations}

Altough the relaxation rates ( $\mathrm{R} 2^{*}$ and $\mathrm{R} 1$ ) are proportional to the tissue iron content, we used their inverses (T2* and $\mathrm{T} 1$ ), since they are commonly adopted in the clinical practice, however an appropriate statistical approach has been used.

White blood sequences were used for $\mathrm{T} 2 *$ assessment, although black blood sequences are more accurate and robust [46].

Conversely to the $\mathrm{T} 2 *$ technique $[9,10], \mathrm{T} 1$ mapping lacks appropriate calibration against histological samples.

Multicenter studies are needed to test the transferability for the T1 mapping as robust and useful complementary tool to $\mathrm{T}_{2} *$ in the clinical practice and to verify the results in populations with lower prevalence of $\mathrm{HCV}$ infection in which a lower frequency of myocardial fibrosis may be attended.

This is a cross-sectional study and prospective studies are recommended to evaluate the predictive value of native $\mathrm{T} 1$ for cardiac outcomes in $\beta$-TM.

\section{Conclusion}

Native T1 is less specific than T2* mapping for MIO detection, given that myocardial fat and diffuse fibrosis can influence T1 values. Conversely, native T1 by a segmental approach can improve the sensitivity in detecting myocardial iron loading and is associated with cardiac complications in $\beta$-TM. The combined use of both T1 and $\mathrm{T} 2 *$ mapping by a segmental approach could therefore improve the detection of mild iron loading, potentially supporting clinicians in appropriately and timely modifying chelation therapy.

\section{Abbreviations}

B-TM: Beta thalassaemia major; Cl: Confidence intervals; CMR: Cardiovascular magnetic resonance; CoV: Coefficient of variation; E-MIOT: 
Extension-myocardial iron overload in thalassaemia; ECG: Electrocardiogram; ECV: Extracellular volume; HF: Heart failure; ICC: Intraclass correlation coefficient; LGE: Late gadolinium enhancement; LV: Left ventricle/left ventricular; MIO: Myocardial iron overload; MOLLI: Modified Look-Locker inversion recovery; OR: Odds ratio; ROC: Receiver operator curve; SD: Standard deviation; TE: Echo time.

\section{Acknowledgements}

We would like to thank all the colleagues involved in the E-MIOT project (https://emiot.ftgm.it/). We thank Claudia Santarlasci for her skillful secretarial work and all patients for their cooperation.

\section{Authors' contributions}

AM analyzed the data, interpreted the results, and wrote the paper. NM and VP were responsible for the setup of the MOLLI sequence and developed the software for image analysis. ADL and SS analyzed native T1 images. LP was responsible for data collection. AS, TC, PPB, MA, and PMGS collected the data. $\mathrm{RDC}$ and GS contributed to the interpretation of the results. AP conceived, designed and supervised the study. All authors contributed to critical revision of the manuscript and read and approved the final version.

\section{Funding}

The E-MIOT project receives "no-profit support" from industrial sponsorships (Chiesi Farmaceutici S.p.A. and ApoPharma Inc.). The funders had no role in study design, data collection and analysis, decision to publish, or preparation of the manuscript.

\section{Availability of data and materials}

The datasets used and/or analysed during the current study are available from the corresponding author on reasonable request.

\section{Declarations}

Ethics approval and consent to participate

The study complied with the Declaration of Helsinki and was approved by the local ethical committee. All patients gave written informed consent.

\section{Consent for publication}

Not applicable.

\section{Competing interests}

$A P$ is the $\mathrm{Pl}$ of the E-MIOT project that receives "no-profit support" from industrial sponsorships (Chiesi Farmaceutici S.p.A., ApoPharma Inc.). AP, AM and LP received speakers' honoraria from Chiesi Farmaceutici S.p.A.. The remaining authors have nothing to disclose.

\section{Author details}

${ }^{1}$ Magnetic Resonance Imaging Unit, Fondazione G. Monasterio CNR-Regione Toscana, Pisa, Italy. ${ }^{2}$ Cardiovascular Department, University of Trieste, Trieste, Italy. ${ }^{3}$ Cardiovascular Division, University of Pisa, Pisa, Italy. ${ }^{4}$ Unità Operativa Semplice Dipartimentale Malattie Rare del Globulo Rosso, Azienda Ospedaliera di Rilievo Nazionale "A. Cardarelli", Napoli, Italy. ${ }^{5}$ Centro Talassemie ed Emoglobinopatie, Ospedale "Meyer", Firenze, Italy. ${ }^{6}$ Servizio Immunoematologia e Medicina Trasfusionale, Dipartimento dei Servizi, Presidio Ospedaliero "San Francesco" ASL Nuoro, Nuoro, Italy. ${ }^{7}$ Ematologia Microcitemia, Ospedale San Giovanni di Dio, ASP Crotone, Crotone, Italy. ${ }^{8}$ Servizio Trasfusionale Aziendale, Azienda Ospedaliero-Universitaria di Sassari, Sassari, Italy.

Received: 4 January 2021 Accepted: 28 April 2021

Published online: 14 June 2021

\section{References}

1. Modell B, Khan M, Darlison M, Westwood MA, Ingram D, Pennell DJ. Improved survival of thalassaemia major in the UK and relation to T2* cardiovascular magnetic resonance. J Cardiovasc Magn Reson. 2008;10(1):42.

2. Pepe A, Meloni A, Pistoia L, Cuccia L, Gamberini MR, Lisi R, et al. MRI multicentre prospective survey in thalassaemia major patients treated with deferasirox versus deferiprone and desferrioxamine. Br J Haematol. 2018;183(5):783-95.

3. Wood JC, Otto-Duessel M, Aguilar M, Nick H, Nelson MD, Coates TD, et al. Cardiac iron determines cardiac $\mathrm{T} 2^{*}, \mathrm{~T} 2$, and $\mathrm{T} 1$ in the gerbil model of iron cardiomyopathy. Circulation. 2005;112(4):535-43.

4. Anderson LJ, Holden S, Davis B, Prescott E, Charrier CC, Bunce NH, et al. Cardiovascular T2-star $\left(\mathrm{T}^{*}\right)$ magnetic resonance for the early diagnosis of myocardial iron overload. Eur Heart J. 2001;22(23):2171-9.

5. Pennell DJ, Udelson JE, Arai AE, Bozkurt B, Cohen AR, Galanello R, et al. Cardiovascular function and treatment in beta-thalassemia major: a consensus statement from the American Heart Association. Circulation. 2013;128(3):281-308

6. Westwood MA, Anderson LJ, Firmin DN, Gatehouse PD, Lorenz CH, Wonke B, et al. Interscanner reproducibility of cardiovascular magnetic resonance $\mathrm{T}^{*}$ measurements of tissue iron in thalassemia. J Magn Reson Imaging. 2003;18(5):616-20.

7. Pepe A, Lombardi M, Positano V, Cracolici E, Capra M, Malizia R, et al. Evaluation of the efficacy of oral deferiprone in beta-thalassemia major by multislice multiecho T2*. Eur J Haematol. 2006;76(3):183-92.

8. Ramazzotti A, Pepe A, Positano V, Rossi G, De Marchi D, Brizi MG, et al. Multicenter validation of the magnetic resonance $t 2 *$ technique for segmental and global quantification of myocardial iron. J Magn Reson Imaging. 2009;30(1):62-8.

9. Carpenter JP, He T, Kirk P, Roughton M, Anderson LJ, de Noronha SV, et al. On T2* magnetic resonance and cardiac iron. Circulation. 2011;123(14):1519-28.

10. Meloni A, Maggio A, Positano V, Leto F, Angelini A, Putti MC, et al. CMR for myocardial iron overload quantification: calibration curve from the MIOT network. Eur Radiol. 2020;29(5):2246-52.

11. Kirk P, Roughton M, Porter JB, Walker JM, Tanner MA, Patel J, et al. Cardiac T2* magnetic resonance for prediction of cardiac complications in thalassemia major. Circulation. 2009;120(20):1961-8.

12. Pepe A, Meloni A, Rossi G, Midiri M, Missere M, Valeri G, et al. Prediction of cardiac complications for thalassemia major in the widespread cardiac magnetic resonance era: a prospective multicentre study by a multi-parametric approach. Eur Heart J Cardiovasc Imaging. 2018;19(3):299-309.

13. Wood JC, Ghugre N. Magnetic resonance imaging assessment of excess iron in thalassemia, sickle cell disease and other iron overload diseases. Hemoglobin. 2008;32(1-2):85-96.

14. Feng Y, He T, Carpenter JP, Jabbour A, Alam MH, Gatehouse PD, et al. In vivo comparison of myocardial T1 with T2 and T2* in thalassaemia major. J Magn Reson Imaging. 2013:38(3):588-93.

15. Sado DM, Maestrini V, Piechnik SK, Banypersad SM, White SK, Flett AS, et al. Noncontrast myocardial T1 mapping using cardiovascular magnetic resonance for iron overload. J Magn Reson Imaging. 2015;41 (6):1505-11.

16. Krittayaphong $R$, Zhang S, Saiviroonporn P, Viprakasit V, Tanapibunpon $P$, Komoltri $C$, et al. Detection of cardiac iron overload with native magnetic resonance T1 and T2 mapping in patients with thalassemia. Int J Cardiol. 2017;248:421-6.

17. Torlasco C, Cassinerio E, Roghi A, Faini A, Capecchi M, Abdel-Gadir A, et al. Role of T1 mapping as a complementary tool to T2* for non-invasive cardiac iron overload assessment. PLoS ONE. 2018;13(2):e0192890.

18. Krittayaphong R, Zhang S, Saiviroonporn P, Viprakasit V, Tanapibunpon $P$, Rerkudom B, et al. Assessment of cardiac iron overload in thalassemia with MRI on 3.0-T: high-field T1, T2, and T2* quantitative parametric mapping in comparison to T2* on 1.5-T. JACC Cardiovasc Imaging. 2019;12(4):752-4.

19. Meloni A, Positano V, Pepe A, Rossi G, Dell'Amico M, Salvatori C, et al. Preferential patterns of myocardial iron overload by multislice multiecho $T^{*} 2$ CMR in thalassemia major patients. Magn Reson Med. 2010;64(1):211-9.

20. Pepe A, Meloni A, Filosa A, Pistoia L, Borsellino Z, D'Ascola DG, et al. Prospective CMR survey in children with thalassemia major: insights from a national network. JACC Cardiovasc Imaging. 2020;13(5):1284-6.

21. Meloni A, Restaino G, Borsellino Z, Caruso V, Spasiano A, Zuccarelli A, et al. Different patterns of myocardial iron distribution by whole-heart T2* magnetic resonance as risk markers for heart complications in thalassemia major. Int J Cardiol. 2014;177(3):1012-9.

22. Pepe A, Positano V, Capra M, Maggio A, Lo Pinto C, Spasiano A, et al. Myocardial scarring by delayed enhancement cardiovascular magnetic resonance in thalassaemia major. Heart. 2009;95:1688-93. 
23. Hanneman K, Nguyen ET, Thavendiranathan P, Ward R, Greiser A, Jolly MP, et al. Quantification of myocardial extracellular volume fraction with cardiac MR imaging in thalassemia major. Radiology. 2016;279(3):720-30.

24. Pepe A, Positano V, Santarelli F, Sorrentino F, Cracolici E, De Marchi D, et al. Multislice multiecho T2* cardiovascular magnetic resonance for detection of the heterogeneous distribution of myocardial iron overload. J Magn Reson Imaging. 2006;23(5):662-8.

25. Meloni A, De Marchi D, Pistoia L, Grassedonio E, Peritore G, Preziosi P, et al. Multicenter validation of the magnetic resonance T2* technique for quantification of pancreatic iron. Eur Radiol. 2019;29(5):2246-52.

26. Messroghli DR, Radjenovic A, Kozerke S, Higgins DM, Sivananthan MU, Ridgway JP. Modified Look-Locker inversion recovery (MOLLI) for highresolution T1 mapping of the heart. Magn Reson Med. 2004;52(1):141-6.

27. Cerqueira MD, Weissman NJ, Dilsizian V, Jacobs AK, Kaul S, Laskey WK, et al. Standardized myocardial segmentation and nomenclature for tomographic imaging of the heart: a statement for healthcare professionals from the Cardiac Imaging Committee of the Council on Clinical Cardiology of the American Heart Association. Circulation. 2002;105(4):539-42.

28. Positano V, Pepe A, Santarelli MF, Scattini B, De Marchi D, Ramazzotti A, et al. Standardized T2* map of normal human heart in vivo to correct T2* segmental artefacts. NMR Biomed. 2007;20(6):578-90.

29. Marsella M, Borgna-Pignatti C, Meloni A, Caldarelli V, Dell'Amico MC, Spasiano A, et al. Cardiac iron and cardiac disease in males and females with transfusion-dependent thalassemia major: a T2* magnetic resonance imaging study. Haematologica. 2011;96(4):515-20.

30. Meloni A, Pepe A, Positano V, Favilli B, Maggio A, Capra M, et al. Influence of myocardial fibrosis and blood oxygenation on heart T2* values in thalassemia patients. J Magn Reson Imaging. 2009;29(4):832-7.

31. Arheden H, Saeed M, Higgins CB, Gao DW, Bremerich J, Wyttenbach $\mathrm{R}$, et al. Measurement of the distribution volume of gadopentetate dimeglumine at echo-planar MR imaging to quantify myocardial infarction: comparison with 99mTc-DTPA autoradiography in rats. Radiology. 1999;211(3):698-708.

32. Jessup M, Abraham WT, Casey DE, Feldman AM, Francis GS, Ganiats TG, et al. 2009 focused update: ACCF/AHA Guidelines for the Diagnosis and Management of Heart Failure in Adults: a report of the American College of Cardiology Foundation/American Heart Association Task Force on Practice Guidelines: developed in collaboration with the International Society for Heart and Lung Transplantation. Circulation. 2009;119(14):1977-2016.

33. Buxton $A E$, Calkins $H$, Callans DJ, DiMarco JP, Fisher JD, Greene $H L$, et al. ACC/AHA/HRS 2006 key data elements and definitions for electrophysiological studies and procedures: a report of the American College of Cardiology/American Heart Association Task Force on Clinical Data Standards (ACC/AHA/HRS Writing Committee to Develop Data Standards on Electrophysiology). Circulation. 2006;114(23):2534-70.

34. Meloni A, Positano V, Ruffo GB, Spasiano A, D'Ascola DG, Peluso A, et al. Improvement of heart iron with preserved patterns of iron store by CMR-guided chelation therapy. Eur Heart J Cardiovasc Imaging. 2015;16(3):325-34.
35. Messroghli DR, Moon JC, Ferreira VM, Grosse-Wortmann L, He T, Kellman $P$, et al. Clinical recommendations for cardiovascular magnetic resonance mapping of $\mathrm{T} 1, \mathrm{~T} 2, \mathrm{~T}_{2}^{*}$ and extracellular volume: a consensus statement by the Society for Cardiovascular Magnetic Resonance (SCMR) endorsed by the European Association for Cardiovascular Imaging (EACVI). J Cardiovasc Magn Reson. 2017;19(1):75.

36. Puntmann VO, Voigt T, Chen Z, Mayr M, Karim R, Rhode K, et al. Native T1 mapping in differentiation of normal myocardium from diffuse disease in hypertrophic and dilated cardiomyopathy. JACC Cardiovasc Imaging. 2013;6(4):475-84

37. Miller CA, Naish JH, Bishop P, Coutts G, Clark D, Zhao S, et al. Comprehensive validation of cardiovascular magnetic resonance techniques for the assessment of myocardial extracellular volume. Circ Cardiovasc Imaging. 2013;6(3):373-83.

38. Positano V, Pepe A, Santarelli MF, Ramazzotti A, Meloni A, De Marchi $D$, et al. Multislice multiecho $T 2^{*}$ cardiac magnetic resonance for the detection of heterogeneous myocardial iron distribution in thalassaemia patients. NMR Biomed. 2009;22(7):707-15.

39. Cheung JS, Au WY, Ha SY, Kim D, Jensen JH, Zhou IY, et al. Reduced transverse relaxation rate (RR2) for improved sensitivity in monitoring myocardial iron in thalassemia. J Magn Reson Imaging. 2011;33(6):1510-6.

40. Rosmini S, Bulluck H, Captur G, Treibel TA, Abdel-Gadir A, Bhuva AN, et al. Myocardial native $\mathrm{T} 1$ and extracellular volume with healthy ageing and gender. Eur Heart J Cardiovasc Imaging. 2018;19(6):615-21.

41. Pepe A, Gamberini MR, Missere M, Pistoia L, Mangione M, Cuccia L, et al. Gender differences in the development of cardiac complications: a multicentre study in a large cohort of thalassaemia major patients to optimize the timing of cardiac follow-up. Br J Haematol. 2018;180(6):879-88.

42. Sado DM, White SK, Piechnik SK, Banypersad SM, Treibel T, Captur G, et al. Identification and assessment of Anderson-Fabry disease by cardiovascular magnetic resonance noncontrast myocardial T1 mapping. Circ Cardiovasc Imaging. 2013;6(3):392-8.

43. Casale M, Meloni A, Filosa A, Cuccia L, Caruso V, Palazzi G, et al. Multiparametric cardiac magnetic resonance survey in children with thalassemia major: a multicenter study. Circ Cardiovasc Imaging. 2015;8(8):e003230.

44. Pepe A, Meloni A, Rossi G, Caruso V, Cuccia L, Spasiano A, et al. Cardiac complications and diabetes in thalassaemia major: a large historical multicentre study. Br J Haematol. 2013;163(4):520-7.

45. Pepe A, Meloni A, Borsellino Z, Cuccia L, Borgna-Pignatti C, Maggio A, et al. Myocardial fibrosis by late gadolinium enhancement cardiac magnetic resonance and hepatitis C virus infection in thalassemia major patients. J Cardiovasc Med (Hagerstown). 2015;16(10):689-95.

46. He T, Gatehouse PD, Kirk P, Tanner MA, Smith GC, Keegan J, et al. Blackblood T2* technique for myocardial iron measurement in thalassemia. J Magn Reson Imaging. 2007;25(6):1205-9.

\section{Publisher's Note}

Springer Nature remains neutral with regard to jurisdictional claims in published maps and institutional affiliations.

\footnotetext{
Ready to submit your research? Choose BMC and benefit from:

- fast, convenient online submission

- thorough peer review by experienced researchers in your field

- rapid publication on acceptance

- support for research data, including large and complex data types

- gold Open Access which fosters wider collaboration and increased citations

- maximum visibility for your research: over $100 \mathrm{M}$ website views per year
}

At BMC, research is always in progress.

Learn more biomedcentral.com/submissions 